\title{
Analysis of Time Delay between Bioimpedance and Respiratory Volume Signals under Inspiratory Loaded Breathing
}

\author{
Dolores Blanco-Almazán ${ }^{1}$, Willemijn Groenendaal ${ }^{2}$, Francky Catthoor ${ }^{3}$, Fellow, IEEE, and \\ Raimon Jané ${ }^{1}$, Senior Member, IEEE
}

\begin{abstract}
Bioimpedance is known for its linear relation with volume during normal breathing. For that reason, bioimpedance can be used as a noninvasive and comfortable technique for measuring respiration. The goal of this study is to analyze the temporal behavior of bioimpedance measured in four different electrode configurations during inspiratory loaded breathing. We measured four bioimpedance channels and airflow simultaneously in $\mathbf{1 0}$ healthy subjects while incremental inspiratory loads were imposed. Inspiratory loading threshold protocols are associated with breathing pattern changes and were used in respiratory mechanics studies. Consequently, this respiratory protocol allowed us to induce breathing pattern changes and evaluate the temporal relationship of bioimpedance with volume. We estimated the temporal delay between bioimpedance and volume respiratory cycles to evaluate the differences in their temporal behavior. The delays were computed as the lag which maximize the cross-correlation of the signals cycle by cycle. Six of the ten subjects showed delays in at least two different inspiratory loads. The delays were dependent on electrode configuration, hence the appearance of the delays between bioimpedance and volume were conditioned to the location and geometry of the electrode configuration. In conclusion, the delays between these signals could provide information about breathing pattern when breathing conditions change.
\end{abstract}

\section{INTRODUCTION}

Bioimpedance is a noninvasive technique capable to measure impedance changes in the body. When bioimpedance is measured in the thorax the impedance changes are due to respiration but also to the cardiac activity which can be removed by signal processing. Previous studies reported the suitability of bioimpedance as a measure of respiration because of its linear relation with volume [1]-[10].

The effects of electrode configurations in bioimpedance signal have been widely studied [4]-[6], [9]. In particular, Seppä et al. reported better bioimpedance performance in the

\footnotetext{
*This work was supported by the Universities and Research Secretariat from the Ministry of Business and Knowledge/Generalitat de Catalunya (FIDGR and GRC 2017 SGR 01770), by the Spanish Ministry of Economy and Competitiveness through the project DPI2015-68820-R (MINECO/FEDER) and by CERCA Programme/Generalitat de Catalunya.

${ }^{1} \mathrm{D}$. Blanco-Almazán and R. Jané are with the Institute for Bioengineering of Catalonia (IBEC), The Barcelona Institute of Science and Technology, 08028 Barcelona Spain, the Department of Automatic Control (ESAII) at the Universitat Politècnica de Catalunya, 08034 Barcelona, Spain and also with the Biomedical Research Networking Center in Bioengineering, Biomaterials and Nanomedicine (CIBER-BBN), 08028, Barcelona, Spain (emails: dblancolibecbarcelona.eu and rjanelibecbarcelona.eu).

${ }^{2}$ W. Groenendaal is with the Holst Centre/imec, Eindhoven 5656AE, The Netherlands (email: Willemijn.Groenendaal@imec-nl.nl).

${ }^{3} \mathrm{~F}$. Catthoor is with imec and KU Leuven, Heverlee 3001, Belgium (email: Francky. Catthoor@imec.be).
}

lateral configuration than in the anteroposterior ones [5] and specifically, De Cannière et al. reported better performance in the configuration located on the midaxillary line. The respiratory protocols used in these studies included free and variable volume but in one study expiratory loads were imposed to the subjects [7]. The objective of imposing expiratory loads was to force changes in the subjects' breathing and the results determined good agreement of bioimpedance to reproduce flow-volume curves. Therefore, it is important to evaluate the performance of bioimpedance in different respiratory conditions. In particular, incremental inspiratory threshold protocols were used in respiratory mechanics studies in healthy subjects and patients and breathing pattern changes associated with this kind of protocol were reported [11], [12]. Based on these two important issues for bioimpedance measurement, our study included different electrode configurations, and different respiratory conditions caused by inspiratory loaded breathing.

The objective of this study was to analyze the temporal behavior of bioimpedance in relation with volume in four electrode configurations during an incremental inspiratory threshold protocol. Temporal differences in bioimpedance electrode configuration could provide additional information related to breathing strategy of the subjects when different breathing conditions are imposed.

\section{MATERIALS AND METHODS}

\section{A. Subjects and Respiratory Protocol}

The test population consisted of 10 healthy subjects (4 females and 6 males) aged 24-37 (mean $=30.5$ and standard deviation $=5.1)$ years and body mass indexes were [19.5 $26.8]$ (mean $=23.1$ and standard deviation $=2.51)$. None were smokers or had reported any respiratory disease. The study was approved by the institutional review board and written consent was obtained from all subjects.

The subjects performed an incremental inspiratory threshold loading protocol during the physiological signal measurements. First, the subjects were asked to perform a maximal respiratory maneuver to get the maximal static inspiratory pressure (MIP). After this maneuver, the quiet breathing (QB) of the subjects was recorded for 2 minutes. The loading part of the protocol consisted of impose inspiratory threshold loads to the subjects. The five inspiratory threshold loads increased progressively from $12 \%$ to $60 \%$. Each load consisted of 30 breaths and a resting time for the subjects to return to baseline before the next load. The subjects were comfortably seated in upright position. The same inspiratory 


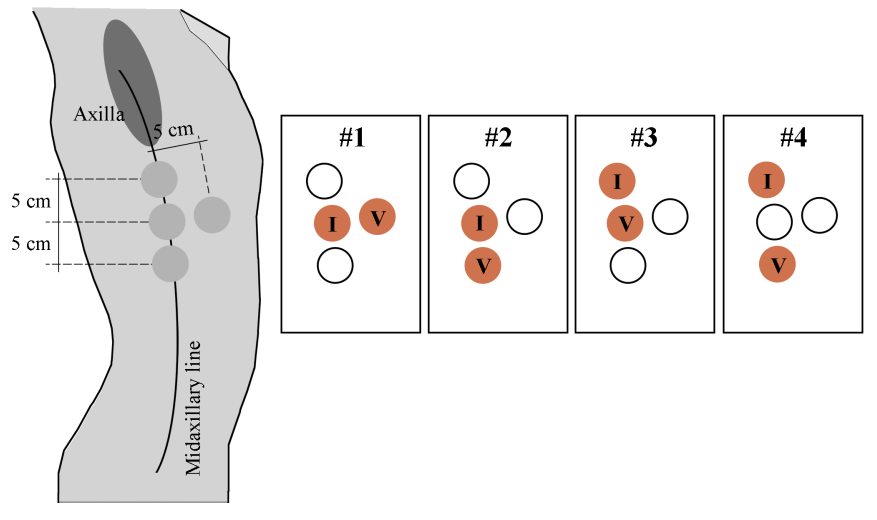

Fig. 1. The four tetra-polar electrode configurations. Only the right side is shown because the configurations were symmetric from the midsternal line. \#1, \#2, \#3 and \#4 denote the electrode configurations where the used electrodes are highlighted, $I$ refers to the injecting electrodes and $V$ to the voltage measurement electrodes.

threshold loading protocol was used before in [13] but to evaluate myographic signals.

\section{B. Equipment}

Airflow, bioimpedance and electrocardigram (ECG) were recorded while the subjects performed the inspiratory threshold loading protocol. The physiological data measurements were made simultaneously by a wearable device and a standard wired research acquisition system.

The wearable device (Stichting imec The Netherlands) was used to measure four bioimpedance channels (Fig. 1) and ECG. The device chip, MUSEIC v1 [14], permits the recording of the bioimpedance channels and ECG simultaneously. The four bioimpedance configurations were tetrapolar with an injection current of $110 \mu \mathrm{A}$ at $80 \mathrm{kHz}$. The use of four electrode configurations allowed us to analyze differences of bioimpedance measurements in different locations and geometries.

Airflow was recorded with Biopac pneumotach transducer (TSD107B, Biopac Systems, Inc., Goleta, CA, USA), amplified 1000 times, filtered by a $300 \mathrm{~Hz}$ analog filter (DA100C, Biopac Systems, Inc.) and A/D converted with MP150 system from Biopac Systems, Inc.

ECG was also recorded using the standard wired system (ECG100C, Biopac Systems, Inc.). Ag/AgCl electrodes were used for bioimpedance and ECG measurements.

An inspiratory muscle trainer (POWERbreathe KH1, POWERbreathe International Ltd, Southam, UK) was used for MIP respiratory maneuver and to impose the inspiratory threshold loads. The subjects breathed through disposable mouth pieces with bacterial filter and wore nasal clips to avoid nasal breathing.

\section{Signal Processing and Delay Estimation}

The signals from both systems were synchronized using cross-correlation from the ECG signals.

Bioimpedance signals were high-pass filtered (zero-phase 8th order Butterworth, $\mathrm{fc}=0.1 \mathrm{~Hz}$ ) and cubic interpolated from sampling frequency of $16 \mathrm{~Hz}$ to $200 \mathrm{~Hz}$.
Airflow signal was low-pass filtered to avoid aliasing and decimated from $10 \mathrm{kHz}$ to $200 \mathrm{~Hz}$. The airflow was lowpass filtered (zero-phase 8th order Butterworth, fc $=0.5 \mathrm{~Hz}$ ) and used to get the volume signal by trapezoidal numerical integration. Volume and bioimpedance signals were low-pass and high-pass filtered (zero-phase 8th order Butterworth, fc $=0.5 \mathrm{~Hz}$ and $\mathrm{fc}=0.1 \mathrm{~Hz}$ respectively).

The delays between volume and bioimpedance signal were computed globally for each load signals and cycle by cycle. The load delays, $\Delta_{L}$ where $L$ is the load number, was estimated as the lag which maximizes the cross-correlation of volume and bioimpedance signals for each load. The cycle delays, $\delta_{i}$ where $i$ is the cycle number, were estimated in the same way as the global ones but cross-correlation was computed for each respiratory cycle individually. The load delays were used to constrain the cycle delays in the range [ $\left.\Delta_{L} \pm N / 4\right], N$ is cycle length. These constraints were needed because contiguous bioimpedance and volume cycles are very similar, and cross-correlation could estimate delays corresponding to the previous or next cycle. Positive delays corresponded to the bioimpedance signal being advanced with respect to the volume whereas negative delays corresponded to the opposite behavior.

The respiratory cycles were selected applying a thresholding algorithm to the airflow signal. The breathing pattern parameters were calculated from the airflow and volume signals measured by a pneumotach. The statistical analysis from these parameters was evaluated by a Mann-Whitney U-test, comparing quiet and inspiratory loaded breathing.

\section{RESULTS}

Airflow and four bioimpedance configurations were recorded at the same time using a wearable device and a standard wired system during an inspiratory threshold protocol in 10 healthy subjects. The delays between bioimpedance channels and volume were estimated using the cross-correlation of the signals cycle by cycle.

Inspiratory loads produces breathing pattern changes with respect to quiet breathing. These changes are summarized in Table I for medium and highest load (36\% and $60 \%$ of subjects' MIP). These parameters were calculated from the airflow and volume signals measured by the pneumotach. In particular, the most significant changes $(p<0.01)$ were in mean inspiratory flow $(M I F)$, inspiratory volume $\left(V_{I}\right)$ and fractional inspiratory time $\left(t_{I} / t_{T}\right)$.

The temporal representation of volume and bioimpedance is shown in Fig. 2 for five different subjects (S01, S02, S05, S08 and S10). The signals correspond to the highest load (60\% of the subject's MIP) and bioimpedance, measured in configuration 4 (see Fig.1). In this figure, S01 is representative of the subjects that did not show delays between the signals. In contrast, subjects S02, S05, S08 and S10 showed notable delays. For these four subjects, the time lag between the signals can be observed in Fig. 2.

The cycle delay evolution over loads is shown in Fig. 3, in this case for subjects S01, S02, S04, S05, S08 and $\mathrm{S} 10$. We chose these subjects as the ones which the delays 
TABLE I

BREATHING PATTERN PARAMETERS DURING QUIET AND LOADED BREATHING

\begin{tabular}{cccc}
\hline Parameter & QB & LOAD 36\% & LOAD 60 \% \\
\hline$M I F(l / s)$ & $0.52 \pm 0.15$ & $1.11 \pm 0.32(*)$ & $1.24 \pm 0.44(*)$ \\
$R R\left(\mathrm{~min}^{-1}\right)$ & $17.07 \pm 3.82$ & $16.30 \pm 5.03$ & $16.35 \pm 5.09$ \\
$t_{I}(s)$ & $1.82 \pm 0.48$ & $1.92 \pm 0.68$ & $1.92 \pm 0.64$ \\
$V_{I}(l)$ & $0.92 \pm 0.28$ & $1.99 \pm 0.55\left(^{*}\right)$ & $2.26 \pm 0.82\left(^{*}\right)$ \\
$t_{I} / t_{T}$ & $0.49 \pm 0.02$ & $0.47 \pm 0.05(*)$ & $0.48 \pm 0.05$ \\
\hline
\end{tabular}

Mean Inspiratory Flow $(M I F)$, Respiratory Rate $(R R)$, Inpiratory Time $\left(t_{I}\right)$, Inspiratory Volume $\left(V_{I}\right)$ and Fractional Inspiratory Time $\left(t_{I} / t_{T}\right)$ for the entire population during quiet breathing $(\mathrm{QB})$, medium and highest load $(36 \%$ and $60 \%)$. (*) p-value $<0.01$ compared with QB

median were different than 0 in at least two loads. For most of these subjects, the delays appeared when inspiratory loads were imposed, specially when the load was high. However, the behavior of the delays differed by subjects, for example, the delays of S04 were higher during the medium loads than during the high ones (Fig 3). In addition, each subject's delays were different depending on configuration as Fig. 3 shows. During the highest load, we observed the delays more frequently in configuration 4 , whereas in the other configurations the delays were more dependent on the subject.

The time lags were higher for S02 which maximum value was $1.775 \mathrm{~s}$, whereas the delays of the other subjects were below $0.7 \mathrm{~s}$. Note that the sign of the delays was also dependent on the subject and configuration.

\section{DISCUSSION}

In this study we analyzed the delays between volume and bioimpedance signals during an incremental inspiratory threshold protocol. The bioimpedance was measured in four tetra-polar electrode configurations simultaneously using a wearable device allowing us to investigate the bioimpedance temporal behavior depending on electrode locations. Delays between volume and bioimpedance were not reported before the present study.

The agreement between bioimpedance and volume was assessed in previous studies [1], [3], [4], [6]. All of these studies reported good agreement between the signals in terms of correlation. Although the agreement between signals was not the goal of this study, we observed good similarity between the signals as Fig. 2 shows. However, none of these studies reported delays between the signals possibly because the protocols under study were different. The respiratory protocols used in these studies included free and variable volume breathing but without any inspiratory loads. In contrast, the respiratory protocol in [7] was based on imposing expiratory loads but their analysis focused on the flow-volume curves and the delays have no influence on those curves.

Inspiratory threshold loading protocols were widely used in studies of respiratory mechanics to assess inspiratory muscle function in healthy subjects and patients. Previous
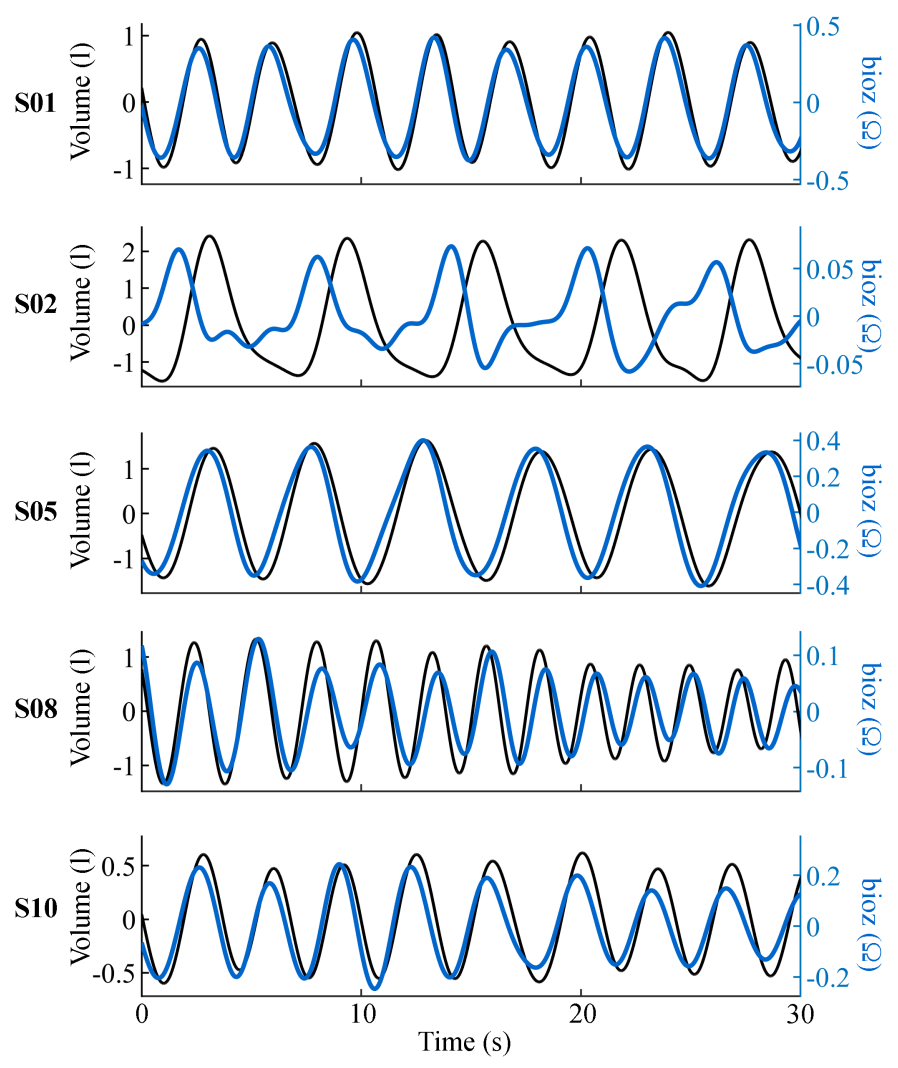

Fig. 2. Temporal representation of volume and bioimpedance (bioz) signals for subjects S01, S02, S05, S08 and S10 during the highest load (60\% of the subjects' MIP) in configuration 4.

respiratory studies used this kind of protocol [11]-[13], [15] and reported breathing pattern changes when incremental inspiratory loads were applied [11], [15]. In our study, we also noticed changes of breathing pattern, specially in the inspiratory volume and mean inspiratory flow parameters (Table I). Eastwood et al. explained that the breathing pattern was coordinate to increase the potency to produce inspiratory force [11]. We hypothesize that the delays between bioimpedance and volume were due to the alteration in shape and motion of the thoracic cage and muscles produced by changes in breathing pattern. In particular, we observed delays with respect to volume in six of the ten subjects (Fig. 3 ). From these six subjects only S04 showed longer delays in medium loads than in the highest ones.

Bioimpedance measurement clearly varies depending on electrode location. Previous studies also used different electrode configurations to evaluate the differences in bioimpedance performance [4]-[6], [9]. Seppä et al. analyzed bioimpedance linearity with volume in different electrode configurations in [4], [6] and reported significant differences. We also observed variability in the delays depending on electrode configurations as Fig. 3 shows. Subjects S02, S05, S08 and S10 exhibited delays in loads $48 \%$ and $60 \%$ in at least two of the vertical configurations $(2,3$ or 4). Configurations 4 was the only one for which the delays appeared for all of these subjects. The consistency 

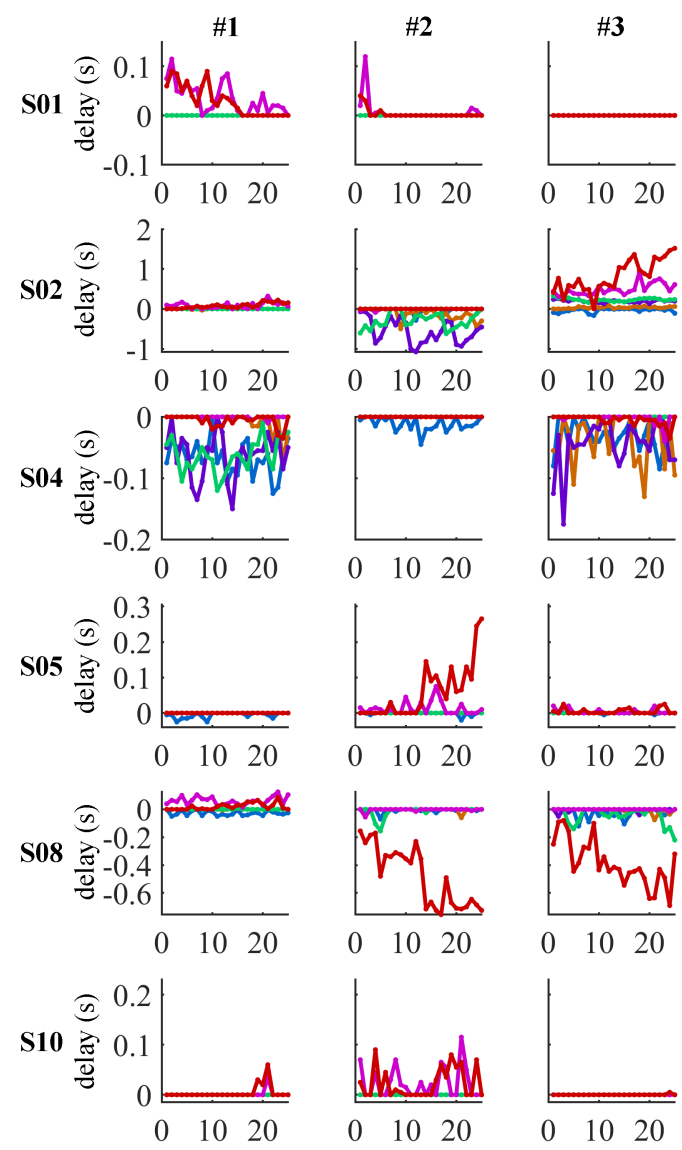

respiratory cycles
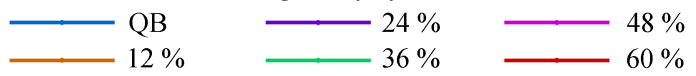

Fig. 3. Cycle delays evolution during quiet breathing (QB) and inspiratory loads for subjects S01, S02, S04, S05, S08 and S10. \#1, \#2, \#3 and \#4 denote the electrode configurations shown in Fig. 1

of this configuration could be because the electrodes of this configuration were the most separated $(10 \mathrm{~cm})$ and covered a broader thoracic zone. The delay behavior in configuration 4 was approximately repeated in other vertical configuration, for subject S02 was the configuration 3, for S05 and S10 was the configuration 2, and for S08 was configurations 2 and 3 . The electrodes of configuration 2 and 3 were equally separated but configuration 2 was lower than configuration 3 . Therefore, we observed that the delays were related to thoracic location and configuration 4 provided more global information. On the contrary, S01 showed delays in the horizontal configuration (1) during the highest loads and not in the vertical ones. Subject S04 was a particular case because the delays appeared in configurations 1 and 3 for different medium loads. Although the agreement with volume remained good in all configurations, configuration 1 can be used to avoid the temporal lags because it was less sensitive to the delays.

The presented results showed that the delays produced in six of the ten subjects were dependent on electrode configuration. Therefore, the delays were related to electrode configuration geometry and thoracic location. In further studies the combination of these four electrode configurations should be analyzed to provide information about the breathing strategies of the subjects when inspiratory loads are applied. We conclude that the delays showed in some subjects could include information associated with the changes in breathing pattern and strategies of the subjects when respiratory conditions are altered, but further validation incorporating other physiological signals is needed.

\section{REFERENCES}

[1] A. Grenvik, S. Ballou, E. McGinley, J. E. Millen, W. L. Cooley, and P. Safar, "Impedance pneumography: Comparison between chest impedance changes and respiratory volumes in 11 healthy volunteers," Chest, vol. 62, no. 4, pp. 439 - 443, 1972.

[2] R. Chen, C. Que, and S. Yan, "Introduction to a new inspiratory threshold loading device," European Respiratory Journal, vol. 12, no. 1, pp. 208-211, 1998.

[3] J. M. Ernst, D. A. Litvack, D. L. Lozano, J. T. Cacioppo, and G. G. Berntson, "Impedance pneumography: Noise as signal in impedance cardiography," Psychophysiology, vol. 36, no. 3, pp. 333-338, 1999.

[4] V.-P. Seppä, J. Viik, A. Naveed, J. Väisänen, and J. Hyttinen, "Signal waveform agreement between spirometer and impedance pneumography of six chest band electrode configurations," in World Congress on Medical Physics and Biomedical Engineering, September 7 - 12, 2009, Munich, Germany, O. Dössel and W. C. Schlegel, Eds. Berlin, Heidelberg: Springer Berlin Heidelberg, 2009, pp. 689-692.

[5] V.-P. Seppä, J. Viik, and J. Hyttinen, "Assessment of pulmonary flow using impedance pneumography," IEEE Transactions on Biomedical Engineering, vol. 57, no. 9, pp. 2277-2285, Sept 2010.

[6] V.-P. Seppä, J. Hyttinen, M. Uitto, W. Chrapek, and J. Viik, "Novel electrode configuration for highly linear impedance pneumography," Biomedizinische Technik/Biomedical Engineering, vol. 58, no. 1, pp. 35-38, jan 2013.

[7] V. Seppä, M. Uitto, and J. Viik, "Tidal breathing flow-volume curves with impedance pneumography during expiratory loading," in 2013 35th Annual International Conference of the IEEE Engineering in Medicine and Biology Society (EMBC), July 2013, pp. 2437-2440.

[8] T. Koivumäki, M. Vauhkonen, J. T. Kuikka, and M. A. Hakulinen, "Bioimpedance-based measurement method for simultaneous acquisition of respiratory and cardiac gating signals," Physiological Measurement, vol. 33, no. 8, p. 1323, 2012.

[9] H. D. Cannière, C. J. P. Smeets, P. M. Vandervoort, S. Lee, G. Squillace, M. Vandecasteele, and L. Grieten, "A wearable bioimpedance device for respiratory monitoring," 2015.

[10] L. P. Malmberg, V.-P. Seppä, A. Kotaniemi-Syrjänen, K. Malmström, M. Kajosaari, A. S. Pelkonen, J. Viik, and M. J. Mäkelä, "Measurement of tidal breathing flows in infants using impedance pneumography," European Respiratory Journal, 2016.

[11] P. R. Eastwood, D. R. Hillman, and K. E. Finucane, "Ventilatory responses to inspiratory threshold loading and role of muscle fatigue in task failure," Journal of Applied Physiology, vol. 76, no. 1, pp. 185-195, 1994, pMID: 8175504.

[12] C. C. Reilly, C. J. Jolley, K. Ward, V. MacBean, J. Moxham, and G. F. Rafferty, "Neural respiratory drive measured during inspiratory threshold loading and acute hypercapnia in healthy individuals," $E x$ perimental Physiology, vol. 98, no. 7, pp. 1190-1198, 2013.

[13] M. Lozano-García, L. Sarlabous, J. Moxham, G. F. Rafferty, A. Torres, R. Jané, and C. J. Jolley, "Surface mechanomyography and electromyography provide non-invasive indices of inspiratory muscle force and activation in healthy subjects," Scientific Reports, vol. 8, no. 1, p. 16921 , dec 2018.

[14] N. V. Helleputte, M. Konijnenburg, H. Kim, J. Pettine, D. Jee, A. Breeschoten, A. Morgado, T. Torfs, H. de Groot, C. V. Hoof, and R. F. Yazicioglu, "18.3 a multi-parameter signal-acquisition soc for connected personal health applications," in 2014 IEEE International Solid-State Circuits Conference Digest of Technical Papers (ISSCC), Feb 2014, pp. 314-315.

[15] F. Laghi, A. Topeli, and M. J. Tobin, "Does resistive loading decrease diaphragmatic contractility before task failure?" Journal of Applied Physiology, vol. 85, no. 3, pp. 1103-1112, 1998, pMID: 9729589. 